\title{
Laboratory-based Evaluation of Wondfo HIV1/ 2 Rapid Test Kits in the Gambia, December 2020
}

\begin{abstract}
Mendy A*, Sanneh ML, J arju ML, Ceesay FB, Kijera M, Rashid M, Suso K, J oof Y, Singhateh A, Saho N, Jah FO, Jallow K, Badjie A, Bayo F, Ceesay M, Sanneh B* and J agne S

National public health laboratories, Ministry of Health, Bertil Harding Highway, Kotu Layout, Kotu, The Gambia *Corresponding author: Bakary Sanneh, National public health laboratories, Ministry of Health, Bertil Harding Highway, Kotu Layout, Kotu, The Gambia

Alphonse Mendy, Bakary Sanneh, National public health laboratories, Ministry of Health, Bertil Harding Highway, Kotu Layout, Kotu, The Gambia
\end{abstract}

Received: August 02, 2021; Accepted: August 30, 2021; Published: September 06, 2021

\section{Introduction}

Acquired Immunodeficiency Syndrome (AIDS) caused by Human Immunodeficiency Virus (HIV) continued to be one of the most important global public health problems. HIV infection gradually compromises and weakens the immune system, which paves way for opportunistic infections like pneumonia and tuberculosis to infect the body and subsequently leading to AIDS [1]. The people living with HIV (PLHIV) globally has reached 38 million in 2019 [2,3]. However, HIV/AIDS related deaths has declined from 1.7 million in 2004 and 1.1 million in 2010 to 690,000 in 2019 With 1.7 million newly infected cases [2]. HIV type 1 (HIV-1) is more common than

\begin{abstract}
Background: HIV rapid diagnosis in The Gambia is mainly done using Determine HIV-1/2 and First Response HIV 1.2.0 or SD Bioline HIV-1/2 3.0 for screening and sero-typing of HIV respectively among children and adults. Polymerase Chain Reaction (PCR) is used for the detection of the HIV viral genome among infants born to HIV positive mothers. This is the HIV testing Algorithm recommended in the HIV testing guidelines of The Gambia for both HIV Counseling and Testing (HCT), Prevention of Mother-to-Child Transmission of HIV (PMTCT) as well as other clinical requests for HIV testing.
\end{abstract}

At the National Public Health Reference Laboratories (NPHRL), ELISA is the first line of testing for HIV while First Response HIV 1.2.0 or SD Bioline HIV $1 / 23.0$ is used for preliminary serotyping. MP Diagnostics HIV Blot 2.2 Western Assay, which is the gold standard for HIV testing in The Gambia, is used as the tie breaker (i.e., when there are discordant results).

The aim of this study was to evaluate the sensitivity and specificity of the Wondfo HIV $1 / 2$ rapid test kit for the detection of human antibodies to HIV in serum/plasma/whole blood and dried blood spots.

Methods: The sensitivity, specificity, positive and negative predictive values of Wondfo HIV $1 / 2$ kits were evaluated in terms of HIV screening against Determine HIV1/2, GenScreen Ultra HIV Ag-Ab ELISA and First Response HIV 1.2.0 using a total of 401 samples. Of these, 351 were sera/plasma samples \{100 HIV negative, 250 HIV positive and 1 indeterminate that were stored at $-20^{\circ} \mathrm{C}, 26$ were whole blood samples (4 negative, 22 positive), and 24 were dried blood spot (DBS) specimens (16 negative and 8 positive) were used for this evaluation. HIV positive specimens were previously sero-typed using First Response HIV-1.2.0 test cards and MP Diagnostics HIV Blot 2.2 Western Assay.

Results: The evaluation shows that the sensitivity, specificity, positive and negative predictive values of Wondfo HIV $1 / 2$ Test Kits with regards to HIV Screening when compared with the GenScreen Ultra HIV Ag-Ab ELISA and First Response $1.2 .0(n=401)$ were $100 \%, 100 \%, 100 \%$, and $100 \%$ respectively. Similarly, the sensitivity, specificity, positive and negative predictive values of Wondfo HIV $1 / 2$ Test Kits with regards to HIV Screening compared to Determine HIV1/2 ( $\mathrm{n}=401)$ were $99.64 \%, 100 \%, 100 \%$, and $99.17 \%$ respectively.

Conclusions: This study demonstrates that Wondfo HIV $1 / 2$ test kits have a high sensitivity and specificity when used for the detection of HIV antibodies using human serum/plasma, whole blood or DBS.

Keywords: HIV; Wondfo; Determine; ELISA; DBS; Laboratory based evaluation; The Gambia
J Bacteriol Mycol - Volume 8 Issue 6 - 2021

ISSN : 2471-0172 | www.austinpublishing group.com

Mendy et al. (C) All rights are reserved type 2 (HIV-2) worldwide and HIV 1 differs from HIV 2 in its higher pathogenicity [4].

The first case of AIDS was discovered in The Gambia in 1986. Since then, the HIV epidemic in The Gambia has never gone beyond 5\%. The demographic and Health Survey (DHS) 2013 results shows that HIV prevalence among adults $15-49$ years in The Gambia is $1.9 \%$ (DHS 2013) [5]. The National Sentinel survey (NSS) among antenatal women showed a declining trend of HIV prevalence from $2.8 \%$ in 2006 to $1.824 \%$ in 2017 [6,7]. However, the Integrated Bio-behavioral study (IBBS) conducted in 2011 have shown a high prevalence (15.9\%) of HIV among female sex workers (FSW) [8]. Such vulnerable 
groups represent important groups for HIV prevention efforts not only because of their own increased HIV infection rates but because, through their clients that may act reservoir for the transmission of HIV, syphilis and other STI to the lower-risk groups in the population of the country [8].

HIV is mainly diagnosed in the Gambia using Determine HIV1/HIV2 and First Response HIV 1.2.0 or SD Bioline HIV 1/2 3.0 for screening and sero-typing of HIV at peripheral laboratories for HIV counseling and testing (HCT), prevention of mother-tochild (PMTCT) transmission of HIV and other clinical requests in healthcare facilities in The Gambia as recommended in the HIV testing management guidelines $[9,10]$. At the National Public Health Laboratories Reference Laboratories, HIV ELISA is used for first line screening while First Response or SD Bioline are used for sero-typing of HIV while HIV Blot 2.2 Western Assay which is the gold standard is used as the tie breaker.

Literature reviews on Wondfo HIV $1 / 2$ has shown that Wondfo HIV $1 / 2$ has a high sensitivity (100\%) and specificity (100\%) with regards to HIV $[11,12]$. However, most of the evaluations did not include dried blood spot specimen.

\section{Objective}

The objective of this study was to evaluate the sensitivity and specificity of the Wondfo HIV 1/2 rapid test kit for the detection of antibodies to HIV in Serum/Plasma/whole blood and DBS on Filter paper

\section{Materials and Methods}

\section{Specimens}

A total of 401 specimens (351 serum/plasma, 26 whole and 24 -dried blood spot) were used in this study. The 351 serum/plasma samples comprised of 100 HIV negatives, 100 HIV 1,100 HIV 2, 50 HIV $1 / 2$ and 1 indeterminate (positive on Determine and negative on Elisa, first response and western blot), the whole blood samples were $22 \mathrm{HIV}$ positives and $4 \mathrm{HIV}$ negatives while the dried blood samples were $16 \mathrm{HIV}$ negatives and $8 \mathrm{HIV}$ positives samples. The serum/ plasma and dried blood spot samples (received for HIV confirmation and EQA) were obtained from Biobank of the reference serology lab of NPHL while the positive whole blood samples were selected from the samples received for HIV CD4 count and the few negative samples were collected from our staff. Human serum/plasma and dried Blood spot (DBS) harvested from whole blood specimens of patients/clients who underwent HCT or PMTCT services at health facilities across the country. These specimens were initially screened for HIV using Determine HIV-1/2 and First Response HIV-1.2,0 or SD Bioline HIV-1/2 3.0 for screening and sero-typing of HIV at peripheral laboratories and were rescreened at the reference Serology laboratory using HIV ELISA for screening while First Response or SD Bioline, and MP Diagnostics HIV Blot 2.2 Western Assay for sero-typing. Storage of the specimens was done at $-20^{\circ} \mathrm{C}$ at the Reference Serology Laboratory of the National Public Health Laboratories (NPHL), The Gambia. The whole blood samples were fresh samples (not stored). Based on the required sample size for this study, specimens were selected at random from the biobank of NPHL, taking into account the integrity of each specimen at the time of selection. Specimens not deemed fit for the purpose of this work were not selected.

\section{Determine HIV-1/2 test}

For the DBS samples, whole blood samples were place on a whatman 903 filter paper and leftover night to dry. Two complete circles from the filter with the Blood was punched/cut and soaked in 250ul Phosphate Buffer Saline (PBS) and incubated for 24 hours.

Each test unit's protective foil cover was removed and labelled with the corresponding patient identification.

$50 \mu \mathrm{L}$ of plasma/serum, whole blood or eluted DBS was applied to the sample pad of each test unit, one drop of chase buffer was added to the sample pad of each test unit that whole blood was added (the one-minute waiting and addition of chase buffer is only for whole blood samples). The test units were then incubated and the test results were read between 15 minutes and one hour after adding the buffer.

\section{Genscreen Ultra HIV Ag-Ab ELISA}

All specimens selected for the evaluation of Wondfo HIV1/2 test kits were screened with Genscreen Ultra HIV Ag-Ab ELISA kit. A specimen distribution and identification plan (worksheet) was generated followed by the preparation of diluted washing solution and conjugate working solution. Using a precision pipette, $25 \mu \mathrm{L}$ of conjugate 1 (R6) was added in wells that are part of the specimen distribution plan. This was subsequently followed by the addition of $75 \mu \mathrm{L}$ of HIV Ag positive control (R5) to well A1, $75 \mu \mathrm{L}$ of $\mathrm{HIV}$ $\mathrm{Ab}$ positive control (R4) to well $\mathrm{B} 1,75 \mu \mathrm{L}$ of HIV negative control (R3) to wells $\mathrm{C} 1, \mathrm{D} 1$ and $\mathrm{E} 1$, and $75 \mu \mathrm{L}$ of each specimen including an in-house control were added to their respective wells as per their positions on the specimen distribution and identification plan. This microplate was sealed with adhesive cover and placed in an incubator for 1 hour at $37^{\circ} \mathrm{C} \pm 1^{\circ} \mathrm{C}$. Following the 1-hour incubation, the adhesive cover was removed and the contents of the plate were washed away with an automated plate washer to get rid of unbound constituents. $100 \mu \mathrm{L}$ of conjugate $2(\mathrm{R} 7 \mathrm{a}+\mathrm{R} 7 \mathrm{~b})$ was dispensed into each well. The microplate was again sealed and incubated for 30 minutes $\left( \pm 4\right.$ minutes) at room temperature $\left(18-30^{\circ} \mathrm{C}\right)$. After this incubation procedure, the microplate was again washed as described above. A substrate solution was prepared and $80 \mu \mathrm{L}$ was dispensed in all the wells. The microplate was subsequently incubated for 30 minutes ( \pm 4 minutes) at room temperature $\left(18-30^{\circ} \mathrm{C}\right)$ followed by the addition of $100 \mu \mathrm{L}$ of stopping solution (R10). The optical density of the microplate was read at $450 \mathrm{~nm}$ using a plate reader. Cut-off values for the tests were determined and results interpreted accordingly. The presence or absence of detectable antigen or antibodies of HIV-1 and/ or HIV-2 was determined by comparing the absorbance measured for each specimen against the calculated cut-off value for the test.

\section{Wondfo HIV1/2 Test}

The kits were equilibrated at room temperature and the test device removed from the foil pouch. The test devices (cassettes) were labelled with the corresponding patient identification and $10 \mu \mathrm{L}$ of plasma/ serum, whole blood or DBS eluate (eluted in phosphate buffered saline i.e. two punch/cut of the circles on whatman 903 filter paper soak in 250ul of the buffered saline) was added into the specimen well A of each cassette followed by the addition of two drops of assay diluent to the buffer well $\mathrm{B}$. the cassette was then incubated at room temperature $\left(10-30^{\circ} \mathrm{C}\right)$ and the test results were interpreted after 15 minutes but less than 30 minutes. 


\section{Data collection and analysis}

Data was collected from HCT and PMTCT laboratory registers at various public health facilities in The Gambia. Statistical analysis was done by using two by two tables for calculating sensitivity, specificity, positive and negative predictive values.

\section{Ethical issues}

All the specimens were used and stored obtained from patients/ clients who were consented to be tested for HIV by the HIV treatment policy and guideline [9]. There patients/clients signed consent forms prior to their specimens being collected at public health facilities in The Gambia. In order to ensure patient confidentiality, no individual names or addresses were used to identify the specimens used in this study.

\section{Results}

\section{Genscreen Ultra HIV Ag-Ab ELISA versus Wondfo HIV1/2}

A total of 121 specimens that were initially found to be HIV negative on the Genscreen Ultra ELISA also tested negative on Wondfo HIV1/2. The specificity of Wondfo HIV1/2 for HIV screening was hence determined against the Genscreen Ultra HIV Ag-Ab ELISA using a two by two table and was found to be $100 \%$ (Figure 1). The negative predictive value for HIV screening was also determined and found to be $100 \%$, showing that the Wondfo HIV $1 / 2$ kits could be reliably used in ruling out HIV among individuals not infected with the virus.

A total of 280 specimens that tested positive on Genscreen Ultra HIV Ag-Ab ELISA were also screened for HIV infection using Wondfo HIV $1 / 2$ kits. These specimens were initially subtyped into HIV-1, HIV-2 and HIV-1\&2 using First Response HIV test cards and Western Blot Assays. All 280 specimens that tested HIV positive on ELISA also tested positive on the Wondfo HIV 1/2 kits (Figure 2), representing a $100 \%$ sensitivity and a $100 \%$ positive predictive value in terms of HIV screening (Figure 1).

\section{Determine HIV1/HIV2 versus Wondfo HIV1/2}

A total of 120 specimens that were initially found to be HIV negative on Determine HIV1/HIV2 also tested negative on Wondfo HIV1/2. The specificity of Wondfo HIV1/2 for HIV screening was hence determined against the Determine HIV1/HIV2 using a two by two table and was found to be $100 \%$ (Figure 3). The negative predictive value for HIV screening was also determined and found to be $99.17 \%$.

A total of 281 specimens that tested positive on Determine HIV1/HIV2 were also screened for HIV infection using Wondfo HIV $1 / 2$ kits. These specimens were initially sero-typed into HIV1, HIV-2 and HIV-1\&2 using First Response HIV 1.2.0 test cards and Western Blot Assays except one (the indeterminate sample) which was only positive on Determine. Among the 281 specimens that tested HIV positive on Determine, 280 tested positive and one (the indeterminate) tested negative on the Wondfo HIV $1 / 2$ kits, representing a $99.64 \%$ sensitivity and a $100 \%$ positive predictive value in terms of HIV screening (Figure 3 ).

\section{Discussion and Conclusions}

HIV/AIDS still poses a threat to the health of individuals worldwide. In The Gambia, HIV counseling and testing (HCT), prevention of mother-to-child transmission of HIV (PMTCT), HIV viral load quantification and Early Infant Diagnosis of HIV( EID) remains a top national health priority. The PMTCT services have been scaled up in the country and the HIV screening is established and efficient. There are 48 PMTCT sites in the country and there are plans to expand the PMTCT sites to 72 PMTCT sites (adding 24 new PMTCT sites).

Literature reviews on Wondfo HIV $1 / 2$ has shown that Wondfo HIV $1 / 2$ has a high sensitivity (100\%) and specificity (100\%) with regards to HIV [11,12] which is in line with the results found in this study with the expectation of the Determine test kit which has a $99.64 \%$ sensitivity. The sample that was positive on Determine was also found to be negative on all other test kits currently used in The Gambia including the Western Blot, which is the gold standard for HIV, testing in The Gambia. Most of the literatures review do not include dried blood spot samples, which is used in some of the laboratories in The Gambia. This study shows that Wondfo HIV1/2 test kits can be used to test HIV samples collected on DBS. The results were $100 \%$ the same in comparison with the test kits used in The Gambia.

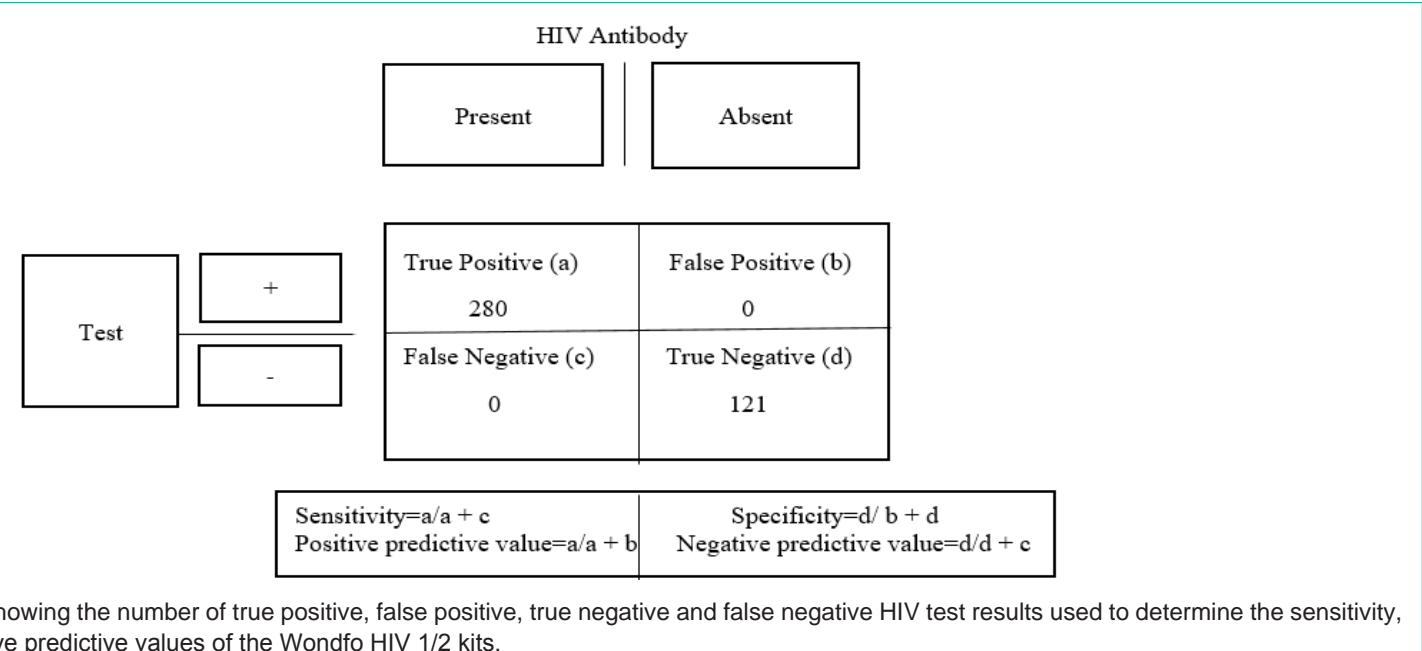

Figure 1: A two by two table showing the number of true positive, false positive, true negative and false negative HIV test results used to determine the sensitivity, specificity, positive and negative predictive values of the Wondfo HIV $1 / 2$ kits. 


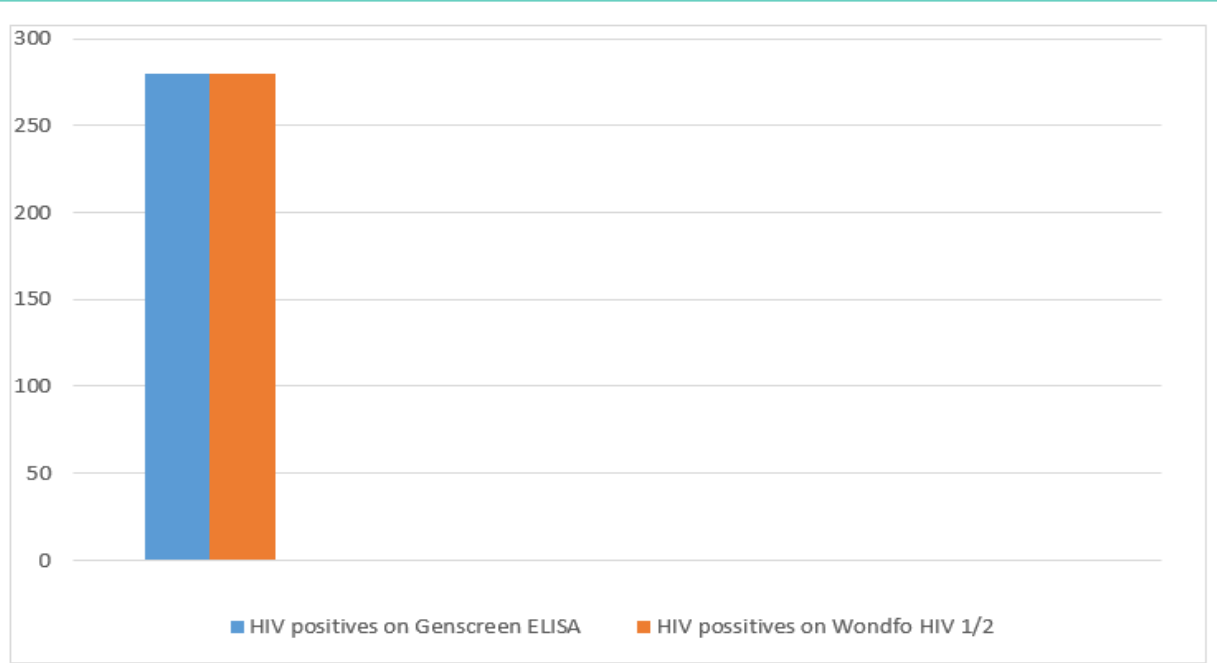

Figure 2: A comparison of Genscreen Ultra HIV Ag-Ab ELISA test results and that of Wondfo HIV $1 / 2$ kits using serum/plasma, whole blood and dried blot spot specimens.

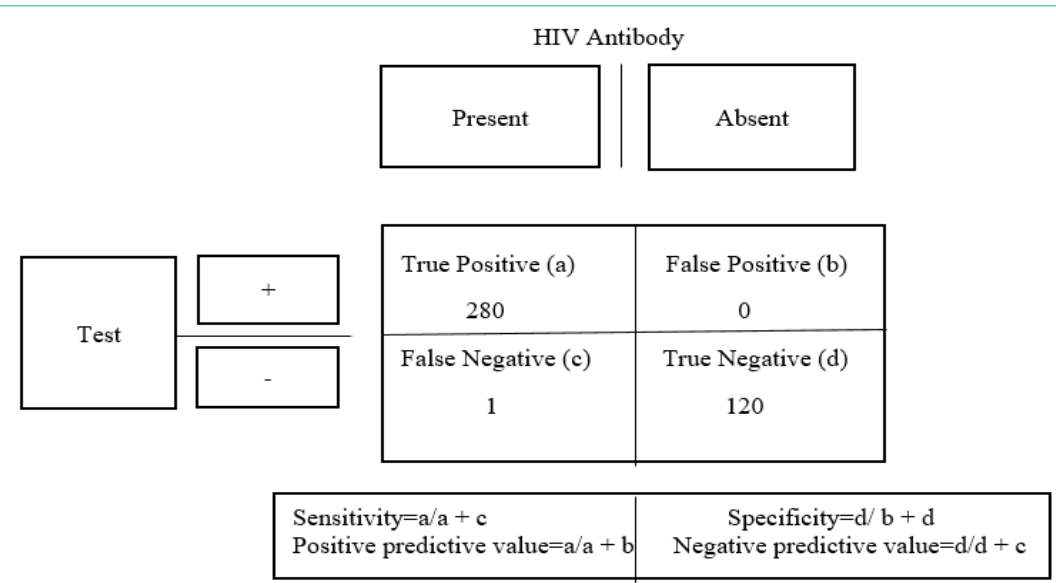

Figure 3: A two by two table showing the number of true positive, false positive, true negative and false negative HIV test results used to determine the sensitivity, specificity, positive and negative predictive values of the Wondfo HIV 1/2 kits.

Expansion of the PMTCT sites is necessary as the prevention and treatment of AIDS depends on prompt and accurate detection of HIV infected patients. In an attempt to attain accuracy in HIV testing, there is a need for the use of highly sensitive and specific test kit, which Determine HIV1/HIV2 is, the current first line screening test kit used in the peripheral laboratories. With reference to this study, Wondfo HIV $1 / 2$ proves to have a high sensitivity and specificity as that of Determine HIV 1.2.0. This implies that Wondfo HIV $1 / 2$ can supplement the use of Determine HIV 1.2.0 which is currently the first line of HIV testing in The Gambia.

\section{Limitations of the Study}

The findings of this study do not represent the diagnostic performance of the Wondfo HIV 1/2 kits using capillary blood, since the specimens used in the study were either serum, EDTA plasma, EDTA whole blood or stored DBS. The biobank of NPHRL was short for indeterminate samples (samples positive on Determine but negative on all other test kits) that is why only one indeterminate sample was included in this evaluation.

\section{Declaration}

Acknowledgements: We would thank the participants from whom these stored samples were from collected and the health care providers from those health facilities. We would also like to thank the Directorate of the NPHL for the technical support and guidance throughout the entire kit evaluation process.

Funding sources: This laboratory-based point of care evaluation project was funded by Ecomed Gambia Ltd. through their collaborative partner.

\section{References}

1. Xihong Zhao, Xiaowei He, Wenmei Li, Liansheng Yang, Jihua Wang. Development and evaluation of a line immunoassay as a confirmatory test for human immunodeficiency virus (HIV)-1/2 infections. African Journal of Biotechnology. 2011; 10: 8434-8439.

2. UNAIDS. Global HIV \& AIDS statistics - 2020 fact sheet. 2020.

3. WHO. HIV data and statistics. 2020.

4. Wang WK, Chen MY, Chuang CY, Jeang KT, Huang LM. Molecular biology of human immunodeficiency virus type 1. J. Microbiol. Immunol. Infect. 2000; 
33: 131-140.

5. GBOS \& ICF. The Gambia Demographic and Health Survey 2013. Banjul, The Gambia and Rockville, Maryland, USA: GBOS and ICF International. 2014.

6. Loeff MF, Sarge-Njie R, Ceesay S, Awasana AA, Jaye P, Sam O, et al Regional differences in HIV trends in The Gambia: results from sentine surveillance among pregnant women. Aids. 2013; 17: 1841-1846.

7. HIV prevalence in The Gambia. Data from the antenatal care sentine surveillance sites 2015-2016. 2016

8. Mason K, Ketende S, Peitzmeier S, Ceesay N, Diouf D, Loum J, et al. A cross-sectional analysis of population demographics, HIV knowledge and risk behaviors, and prevalence and associations of HIV among men who have sex with men in The Gambia. AIDS research and human retroviruses. 2013, 29: $1547-1552$
9. Ministry of Health \& Social Welfare, Republic of The Gambia. Guidelines for Antiretroviral Therapy for the Prevention and Treatment of HIV in The Gambia. Banjul, The Gambia. 2015.

10. Aids, National, Secretariat Office, and the President. "The Republic of The Gambia National Strategic Plan for HIV and AIDS. 2014.

11. Report, WPP 2018. Product: One Step HIV1/2 Whole Blood/Serum/Plasma Test WHO reference number: PQDx 0357-004-00.

12. UNICEF. One Step HIV1/2 WB/Serum/PIsm Test, kit/25. 2018. 